\title{
Optimal Channel Switching for Average Capacity Maximization in the Presence of Switching Delays
}

\author{
Ahmet Dundar Sezer and Sinan Gezici \\ Department of Electrical and Electronics Engineering, Bilkent University, 06800, Ankara, Turkey \\ Emails: adsezer@ee.bilkent.edu.tr, gezici@ee.bilkent.edu.tr
}

\begin{abstract}
In this study, the optimal channel switching problem is investigated for average capacity maximization in the presence of channel switching delays. First, the optimal strategy is obtained and the corresponding average capacity is derived when channel switching is performed among a given number of channels. Then, it is proved that channel switching among more than two different channels is not optimal. Also, the maximum average capacity achieved by the optimal channel switching strategy is expressed as a function of the channel switching delay parameter and the average and peak power limits. Then, scenarios in which the optimal strategy corresponds to the use of a single channel or to channel switching between two channels are described. Numerical examples are presented for showing the effects of channel switching delays.
\end{abstract}

Index Terms-Channel switching, capacity, switching delay, time sharing.

\section{INTRODUCTION}

Optimal resource allocation is of critical importance for improving performance of communication systems. The channel capacity is one of the common metrics that is optimized in resource allocation problems [1]. In [2], the optimal dynamic resource allocation is studied for fading broadcast channels with the consideration of code division, time division, and frequency division in the presence of perfect channel side information at the transmitter and receivers. In [3], an adaptive resource allocation method is presented for multiuser orthogonal frequency division multiplexing (OFDM) systems in the presence of proportional fairness constraints among users, and optimal and suboptimal algorithms are provided for the maximization of sum capacity under constraints on the minimum required data rate for each user.

Optimal time sharing and power allocation can be implemented together for improving the performance of communication systems in which multiple channels between a transmitter and a receiver are available [4], [5]-[9]. In other words, the use of each channel with a certain fraction of time and a certain power level, which is called the channel switching, can be optimized for enhancing the performance of a communication system. The study in [8] investigates the optimal channel switching problem over Gaussian channels under average power and cost constraints. In particular, a certain utilization cost is assigned to each channel, and the average probability of error is minimized in the presence of an average cost constraint. In [9], the optimal channel switching strategy is proposed for average capacity maximization, and it is presented that the optimal strategy can be achieved by channel switching between at most two different channels.
In most of the studies related to optimal channel switching strategies, delays (costs) associated with the channel switching operation are omitted or assumed to be negligible due to improved hardware technologies [4], [5]-[9]. However, due to hardware limitations, the channel switching operation takes a certain time in practice. In particular, when switching to a new channel, the parameters at the transmitter and the receiver are set according to the characteristics (i.e., frequency) of the new channel, which induces a channel switching delay and consequently reduces the available time for data transmission [10], [11]. As investigated in [12], the state-of-the-art algorithms related to scheduling in wireless mesh networks experience performance degradation in the presence of the channel switching latency.

Although the channel switching problem has been investigated from various perspectives, no studies in the literature have considered channel switching for average capacity maximization in the presence of channel switching delays. In this study, the optimal channel switching strategy is proposed for average capacity maximization under power constraints and considering a time delay for each channel switching operation during which data communication cannot be performed. It is observed that consideration of channel switching delays leads to significant differences in the formulation and analyses compared to those obtained by omitting the effects of channel switching delays [9].

The main contributions of this study can be summarized as follows:

- The channel switching problem for average capacity maximization in the presence of channel switching delays is studied for the first time in the literature.

- An alternative optimization problem, which facilitates theoretical investigations, is formulated in terms of the number of channels employed in the channel switching process (Proposition 1 and Proposition 2).

- When the channel switching is to be performed among a certain number of channels, the optimal strategy and the corresponding average capacity are derived (Proposition 3).

- It is shown that channel switching among more than two different channels is not optimal, and an expression for the maximum average capacity of the optimal channel switching strategy is presented (Proposition 4).

\section{System Model And Problem Formulation}

Consider a communication system in which $K$ different channels are available in the communication link between 


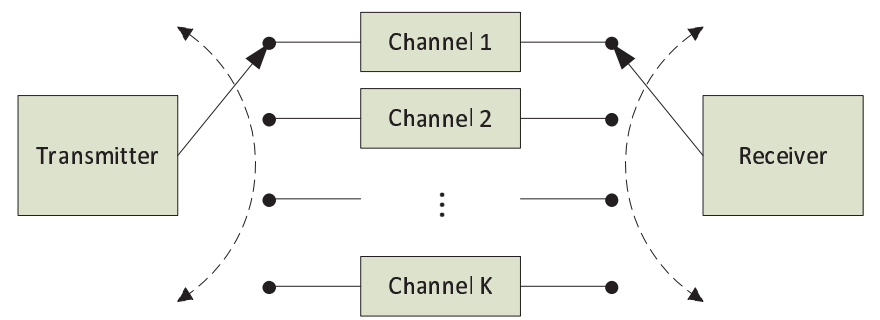

Fig. 1. Block diagram of a communication system in which transmitter and receiver can switch among $K$ channels.

a transmitter and a receiver. The channels are assumed to introduce independent additive Gaussian noise with constant spectral density levels over the channel bandwidths. It is assumed that the spectral density levels and the bandwidths of the channels can be different in general. The transmitter and the receiver can switch among these $K$ channels in order to enhance the capacity of the communication system. At any given time, only one channel can be utilized for the transmission and the transmitter informs the receiver about which channel is occupied for the given time so that the transmitter and the receiver are synchronized [9]. Fig. 1 illustrates the system with $K$ different channels with possibly various bandwidths and noise levels.

Before data communication commences, the transmitter determines a channel switching strategy that will be employed during a time duration of $T_{d}$ seconds and informs the receiver about the channels to be utilized and the respective utilization times according to that strategy. It is assumed that the channel characteristics do not change during $T_{d}$ seconds. To start data communication, the transmitter and the receiver set their parameters for the first channel to be utilized (i.e., they switch to the same channel), and this process is assumed to take a time duration $T_{c s}$ seconds, which is called the channel switching delay (cost). During $T_{c s}$ seconds, there is no data communication and consequently no power is transmitted. Then, data transmission starts and lasts for a certain time duration based on the employed strategy. Next, the transmitter and the receiver switch to the second channel to be utilized, which again takes $T_{c s}$ seconds, and then data communication occurs over that channel for a specified time. The process continues in this manner according to the employed channel switching strategy, which may utilize a subset of all channels in general. For the next period of $T_{d}$ seconds, the optimal channel switching strategy is calculated again according to the new channel characteristics, and communication continues in the same fashion as described above.

In Fig. 2, a sample time frame structure is presented for channel switching over 4 channels. In this case, the transmitter and the receiver communicate during $3 T_{d}$ seconds. In first $T_{d}$ seconds, the channel switching strategy is to communicate over channel 1 and channel 3 for $T_{1}^{1}$ and $T_{3}^{1}$ seconds, respectively, where $T_{1}^{1}+T_{3}^{1}=T_{d}$. Before the data transmission over each channel, there exists a channel switching time (cost) of $T_{c s}$ seconds, which is required for the transmitter and the receiver to set their parameters for communication over the desired channel. During the second $T_{d}$ seconds, the communication is performed over only channel 2 for a time duration of $T_{2}^{2}$ seconds, where $T_{2}^{2}=T_{d}$, and there is no channel switching to another channel in this case. Finally, channels 1,2 and 3 are utilized for the communication in the last $T_{d}$ seconds. It is important to note that it is not necessary to utilize all the channels in a given channel switching strategy. For example, channel 4 is not utilized in any of the channel switching strategies in Fig. 2.

Let $B_{i}$ and $N_{i} / 2$ denote, respectively, the bandwidth and the constant power spectral density level of the additive Gaussian noise for channel $i$, where $i \in\{1, \ldots, K\}$. Then, the capacity of channel $i$ is expressed as

$$
C_{i}(P)=B_{i} \log _{2}\left(1+\frac{P}{N_{i} B_{i}}\right) \text { bits/sec }
$$

where $P$ represents the average transmit power [13].

The main aim of this study is to characterize the optimal channel switching strategy that maximizes the average capacity of the communication system in Fig. 1 under average and peak power constraints and in the presence of channel switching delays. To that aim, channel time-sharing (channel switching) factors are expressed as $\lambda_{1} \triangleq \frac{T_{1}}{T_{d}}, \ldots, \lambda_{K} \triangleq \frac{T_{K}}{T_{d}}$, where $T_{i}$ denotes the amount of time channel $i$ is utilized and $T_{d}$ is the duration over which the channel switching strategy is employed. In addition, $\varepsilon \triangleq \frac{T_{c s}}{T_{d}}$ is defined as the channel switching delay factor, and $\left(\lambda_{i}-\varepsilon\right) \mathbb{1}_{\left\{\lambda_{i}>0\right\}}$ represents the fraction of time when channel $i$ is used for communication, where $\mathbb{1}_{\left\{\lambda_{i}>0\right\}}$ denotes the indicator function, which is equal to 1 if $\lambda_{i}>0$ and 0 otherwise. Then, the following optimal channel switching problem is proposed for capacity maximization in the presence of channel switching delays:

$$
\begin{aligned}
\max _{\left\{\lambda_{i}, P_{i}\right\}_{i=1}^{K}} & \sum_{i=1}^{K} \mathbb{1}_{\left\{\lambda_{i}>0\right\}}\left(\lambda_{i}-\varepsilon\right) C_{i}\left(P_{i}\right) \\
\text { subject to } & \sum_{i=1}^{K} \mathbb{1}_{\left\{\lambda_{i}>0\right\}}\left(\lambda_{i}-\varepsilon\right) P_{i} \leq P_{\mathrm{av}}, \\
& P_{i} \in\left[0, P_{\mathrm{pk}}\right], \forall i \in\{1, \ldots, K\}, \\
& \sum_{i=1}^{K} \lambda_{i}=1, \lambda_{i} \in\{0\} \cup[\varepsilon, 1], \forall i \in\{1, \ldots, K\}
\end{aligned}
$$

where $C_{i}\left(P_{i}\right)$ is as in (1), $P_{i}$ is the average transmit power allocated to channel $i, P_{\mathrm{pk}}$ denotes the peak power limit, and $P_{\mathrm{av}}$ represents the average power limit for the transmitter. It is assumed that $P_{\mathrm{av}}<P_{\mathrm{pk}}$ and $0<\varepsilon<1$. From (2), it is noted that due to the channel switching delay, a channel can be utilized only if its time-sharing factor is larger than or equal to the channel switching delay factor, $\varepsilon$. In addition, $\varepsilon$ fractions are subtracted from both the average capacity and the average power terms since no data transmission occurs during channel switching.

\section{Optimal Channel Switching With Switching DELAYS}

In its current form, the optimization problem in (2) is difficult to solve in general since it is not a convex optimization 


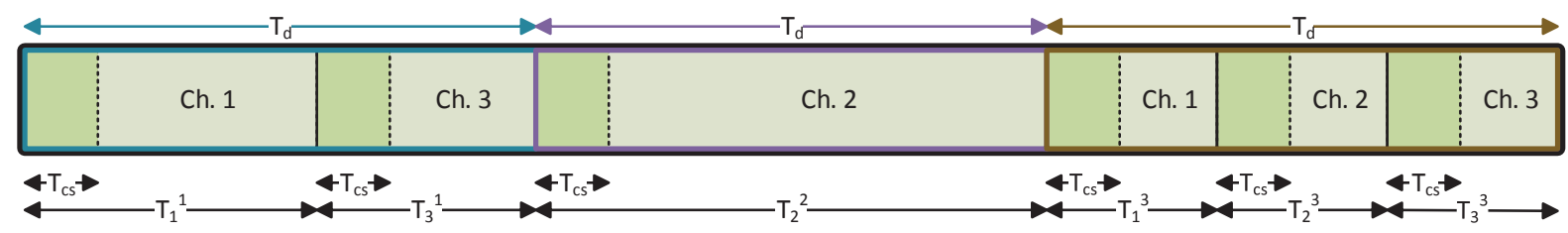

Fig. 2. A sample time frame structure of a communication system in which transmitter and receiver can switch among 4 channels.

problem and requires a search over a $2 K$ dimensional space. Therefore, our aim is to derive an equivalent formulation of the problem in (2), which leads to a low-complexity solution for the optimal channel switching strategy. In the following proposition, this alternative optimization problem is presented. (The proofs of the propositions are not presented due to the space limitation.)

Proposition 1: Define set $A$ as $A=\{1, \ldots, K\}$ and let $\mathcal{P}(A)$ denote the power set of set $A$. Then, the solution of the following optimization problem results in the same maximum value that is achieved by the problem in (2):

$$
\begin{aligned}
& \max _{\tilde{K} \in A} \max _{S \in B^{\tilde{K}}} \max _{\left\{\nu_{s_{i}}, P_{s_{i}}\right\}_{i=1}^{\tilde{K}}} \sum_{i=1}^{\tilde{K}}\left(\nu_{s_{i}}-\varepsilon\right) C_{s_{i}}\left(P_{s_{i}}\right) \\
& \text { subject to } \sum_{i=1}^{\tilde{K}}\left(\nu_{s_{i}}-\varepsilon\right) P_{s_{i}} \leq P_{\mathrm{av}} \\
& P_{s_{i}} \in\left[0, P_{\mathrm{pk}}\right], \forall i \in\{1, \ldots, \tilde{K}\} \\
& \sum_{i=1}^{\tilde{K}} \nu_{s_{i}}=1, \nu_{s_{i}} \geq \varepsilon, \forall i \in\{1, \ldots, \tilde{K}\}
\end{aligned}
$$

where $s_{i}$ represents the ith element of set $S$, and $B^{\tilde{K}}$ is defined as

$$
B^{\tilde{K}} \triangleq\{\chi \in \mathcal{P}(A)|| \chi \mid=\tilde{K}\}
$$

for $\tilde{K} \in\{1, \ldots, K\}$, with $|\chi|$ denoting the cardinality of set $\chi$.

In the optimization problem in (3), parameter $\tilde{K}$ indicates the number of employed channels in a channel switching strategy; that is, the optimization is performed for all possible numbers of employed channels explicitly. In this way, the indicator functions in (2) are removed. Since there exist $K$ available channels in the system, the optimization problem in (3) requires a search over all possible values of $\tilde{K} \in A$, where $A=\{1, \ldots, K\}$. For each $\tilde{K}$, set $B^{\tilde{K}}$ in (4) consists of the sets that are subsets of set $A$ with $\tilde{K}$ elements; that is, $B^{\tilde{K}}$ corresponds to all possible $\tilde{K}$ combinations of $K$ different channels. Hence, $B^{\tilde{K}}$ consists of $\left(\begin{array}{c}K \\ \tilde{K}\end{array}\right)$ sets. For example, if $K=3$ and $\tilde{K}=2$, then $B^{\tilde{K}}=\{\{1,2\},\{1,3\},\{2,3\}\}$. For each element of $B^{\tilde{K}}$, which is denoted by $S$ in (3), the optimization is performed over $\left\{\nu_{s_{i}}, P_{s_{i}}\right\}_{i=1}^{\tilde{K}}$, where $s_{i}$ selects the $i$ th channel in $S$ and $\nu_{s_{i}}$ and $P_{s_{i}}$ denote, respectively, the time-sharing factor and the average transmit power allocated to channel $s_{i}$; i.e., the $i$ th employed (selected) channel.

The optimization problem in (3) is not only more convenient than the one in (2), which involves indicator functions, but also leads to simpler formulations of the optimal channel switching problem. To that end, the following proposition provides a scaled and more compact version of the optimization problem in (3).

Proposition 2: The optimization problem in (3) can be expressed in the form of the following optimization problem:

$$
\begin{aligned}
& \max _{\tilde{K} \in A} \max _{S \in B^{\tilde{K}}} \max _{\left\{\mu_{s_{i}}, P_{s_{i}}\right\}_{i=1}^{\tilde{K}}}(1-\tilde{K} \varepsilon) \sum_{i=1}^{\tilde{K}} \mu_{s_{i}} C_{s_{i}}\left(P_{s_{i}}\right) \\
& \text { subject to } \sum_{i=1}^{\tilde{K}} \mu_{s_{i}} P_{s_{i}} \leq \frac{P_{\mathrm{av}}}{(1-\tilde{K} \varepsilon)} \\
& P_{s_{i}} \in\left[0, P_{\mathrm{pk}}\right], \forall i \in\{1, \ldots, \tilde{K}\} \\
& \sum_{i=1}^{\tilde{K}} \mu_{s_{i}}=1, \mu_{s_{i}} \geq 0, \forall i \in\{1, \ldots, \tilde{K}\} \\
& \tilde{K}<\frac{1}{\varepsilon}
\end{aligned}
$$

where $A, B^{\tilde{K}}$, and $s_{i}$ are as defined in Proposition 1.

The optimization problem in (5) can be separated into two optimization problems based on the value of $\tilde{K}$ as follows:

- Case-1 (Single Channel): For the case in which a single channel is employed for communication, that is, $\tilde{K}=1$, the optimization problem in (5) can be stated as follows:

$$
\begin{aligned}
\max _{S \in B^{1}} \max _{\mu_{s_{1}}, P_{s_{1}}} & (1-\varepsilon) \mu_{s_{1}} C_{s_{1}}\left(P_{s_{1}}\right) \\
\text { subject to } & \mu_{s_{1}} P_{s_{1}} \leq \frac{P_{\mathrm{av}}}{(1-\varepsilon)} \\
& P_{s_{1}} \in\left[0, P_{\mathrm{pk}}\right] \\
& \mu_{s_{1}}=1, \mu_{s_{1}} \geq 0 \\
& \varepsilon<1
\end{aligned}
$$

where $B^{1}=\{\{1\},\{2\}, \ldots,\{K\}\}$ and $s_{1}$ denotes the (first) element of $S$. The optimization problem in (6) is easily solvable by using simple algebra. Let $C_{\mathrm{scs}}$ denote the solution of (6). Then, the achieved maximum capacity via the optimal single channel strategy can be expressed as

$$
C_{\mathrm{scs}}=\max _{l \in\{1, \ldots, K\}}(1-\varepsilon) C_{l}\left(\min \left\{\frac{P_{\mathrm{av}}}{(1-\varepsilon)}, P_{\mathrm{pk}}\right\}\right)
$$

and the channel index $m$ employed in this strategy can be obtained as

$$
m=\underset{l \in\{1, \ldots, K\}}{\arg \max } C_{l}\left(\min \left\{\frac{P_{\mathrm{av}}}{(1-\varepsilon)}, P_{\mathrm{pk}}\right\}\right) .
$$

In the optimal single channel strategy, it is optimal to use 
all the available and attainable power, $\min \left\{\frac{P_{\mathrm{av}}}{(1-\varepsilon)}, P_{\mathrm{pk}}\right\}$ over a single channel since $C_{i}(P)$ in (1) is a monotone increasing and continuous function.

- Case-2 (Channel Switching): Consider the optimization problem in (5) in the presence of channel switching; that is, $\tilde{K} \geq 2$. Then, the following optimization problem is obtained:

$$
\begin{aligned}
C_{\mathrm{css}}= & \max _{\tilde{K} \in A \backslash\{1\}} \max _{S \in B^{\tilde{K}}} \max _{\left\{\mu_{s_{i}}, P_{s_{i}}\right\}_{i=1}^{\tilde{K}}}(1-\tilde{K} \varepsilon) \sum_{i=1}^{\tilde{K}} \mu_{s_{i}} C_{s_{i}}\left(P_{s_{i}}\right) \\
\text { subject to } & \sum_{i=1}^{\tilde{K}} \mu_{s_{i}} P_{s_{i}} \leq \frac{P_{\mathrm{av}}}{(1-\tilde{K} \varepsilon)} \\
& P_{s_{i}} \in\left[0, P_{\mathrm{pk}}\right], \forall i \in\{1, \ldots, \tilde{K}\} \\
& \sum_{i=1}^{\tilde{K}} \mu_{s_{i}}=1, \mu_{s_{i}} \geq 0, \forall i \in\{1, \ldots, \tilde{K}\} \\
& \tilde{K}<\frac{1}{\varepsilon}
\end{aligned}
$$

Based on Case-1 and Case-2, the solution of (5) corresponds to either the single channel strategy or the channel switching strategy. Let $C_{\mathrm{scs}}$ and $C_{\mathrm{css}}$ denote the solutions of the optimization problems in (7) and (9), respectively. Then, the solution of (5) can be calculated as

$$
\max \left(C_{\mathrm{scs}}, C_{\mathrm{css}}\right) \text {. }
$$

As discussed in Case-1, the optimal single channel strategy has a simple closed-form solution. However, it is difficult to solve the channel switching problem in the form of (9). Therefore, the following proposition is presented to simplify the optimization problem in (9).

Proposition 3: Assume that $\bar{K} \geq 2$ channels are employed in the channel switching strategy and $\varepsilon<1 / \bar{K}$ holds. Then, the maximum average capacity achieved via the optimal channel switching strategy over $\bar{K}$ channels can be expressed as

$$
\psi(\bar{K})=\left\{\begin{array}{cc}
\max _{\tilde{P}_{1} \in\left[\frac{P_{\mathrm{av}}}{1-K \varepsilon}, P_{\mathrm{pk}}\right]}(1-\bar{K} \varepsilon)\left(\frac{\frac{P_{\mathrm{av}}}{1-K \varepsilon}-\tilde{P}_{2}}{\tilde{P}_{1}-\tilde{P}_{2}} C_{\max }\left(\tilde{P}_{1}\right)\right. \\
\left.\quad+\frac{P_{\mathrm{av}}}{1-K \varepsilon}\right) \\
\left.+\frac{\tilde{P}_{1}-\frac{P_{\mathrm{av}}}{1-K \varepsilon}}{\tilde{P}_{1}-\tilde{P}_{2}} C_{\max }\left(\tilde{P}_{2}\right)\right), & \text { if } \frac{P_{\mathrm{av}}}{1-K \varepsilon}<P_{\mathrm{pk}} \\
(1-\bar{K} \varepsilon) C_{\max }\left(P_{\mathrm{pk}}\right), & \text { otherwise }
\end{array}\right.
$$

where $C_{\max }(P)$ is defined as

$$
C_{\max }(P) \triangleq \max \left\{C_{1}(P), \ldots, C_{K}(P)\right\} .
$$

Proposition 3 provides a significant simplification for the solution of the optimization problem in (9) and leads to the following formulation for the optimal channel switching strategy (Case-2):

$$
\max _{\tilde{K} \in A \backslash\{1\}} \psi(\tilde{K}) \text { subject to } \tilde{K}<\frac{1}{\varepsilon}
$$

where $\psi(\tilde{K})$ is as in (11). Compared to (9), the problem in (13) has significantly lower computational complexity since its search space is only two-dimensional for each feasible $\tilde{K}$ (see (11)) whereas a search over a $2 \tilde{K}$ dimensional space is required in (9) for each $(\tilde{K}, S)$ pair.

In the following proposition, a general solution for (13) is provided, and it is shown that the optimal channel switching strategy (Case-2) corresponds to switching between two of the channels.

Proposition 4: The optimal channel switching strategy (Case-2) is to switch between two channels; that is, switching among more than two channels is not optimal. In addition, the maximum average capacity $C_{\mathrm{css}}$ achieved by the optimal channel switching strategy, which is obtained as the solution of (13), can be expressed as follows.

$$
C_{\mathrm{cSs}}= \begin{cases}0, & \text { if } \varepsilon \geq \frac{1}{2} \\ (1-2 \varepsilon) C_{\mathrm{max}}\left(P_{\mathrm{pk}}\right), & \text { if } \varepsilon<\frac{1}{2} \text { and } \frac{P_{\mathrm{av}}}{1-2 \varepsilon} \geq P_{\mathrm{pk}} \\ \max _{\tilde{P}_{1} \in\left[\frac{P_{\mathrm{av}}}{1-2 \varepsilon}, P_{\mathrm{pk}}\right]}(1-2 \varepsilon)\left(\frac{\frac{P_{\mathrm{av}}-\tilde{P}_{2}}{1-2 \varepsilon} \tilde{P}_{1}-\tilde{P}_{2}}{\tilde{P}_{2} \in\left[0, \frac{P_{\mathrm{av}}}{1-2 \varepsilon}\right)}\left(\tilde{P}_{1}\right)\right. \\ \left.+\frac{\tilde{P}_{1}-\frac{P_{\mathrm{av}}}{1-2 \varepsilon}}{\tilde{P}_{1}-\tilde{P}_{2}} C_{\max }\left(\tilde{P}_{2}\right)\right), & \text { otherwise }\end{cases}
$$

Based on Proposition 4, the optimal channel switching strategy can be specified in various scenarios. For the first scenario in (14), i.e., for $\varepsilon \geq 1 / 2, C_{\mathrm{css}}=0$ since channel switching is not feasible, as noted from the constraint in (13). For $\varepsilon<1 / 2$ and $P_{\mathrm{av}} /(1-2 \varepsilon) \geq P_{\mathrm{pk}}$, the solution of the optimal channel switching problem is to transmit at power level $P_{\mathrm{pk}}$ over the best channel (that achieves the maximum capacity for power level $P_{\mathrm{pk}}$ ) for a time fraction of $(1-2 \varepsilon)$, then switching to another channel without transmitting any power (i.e., by consuming a time fraction of $\varepsilon$ ), which results in $C_{\mathrm{css}}=(1-2 \varepsilon) C_{\max }\left(P_{\mathrm{pk}}\right)$. Finally, for $\varepsilon<1 / 2$ and $P_{\mathrm{av}} /(1-2 \varepsilon)<P_{\mathrm{pk}}$, the achieved maximum average capacity can be calculated based on (14) as $C_{\text {css }}=(1-2 \varepsilon)\left(\mu^{*} C_{\max }\left(\tilde{P}_{1}^{*}\right)+\left(1-\mu^{*}\right) C_{\max }\left(\tilde{P}_{2}^{*}\right)\right)$, where $\tilde{P}_{1}^{*}$ and $\tilde{P}_{2}^{*}$ are the optimizers of the maximization problem in (14),

$$
\mu^{*}=\left(\frac{P_{\mathrm{av}}}{1-2 \varepsilon}-\tilde{P}_{2}^{*}\right) /\left(\tilde{P}_{1}^{*}-\tilde{P}_{2}^{*}\right),
$$

and the optimal channel switching strategy is to switch between channel $i$ and channel $j$ with power levels $\tilde{P}_{1}^{*}$ and $\tilde{P}_{2}^{*}$, respectively, where $i$ and $j$ are given by ${ }^{1}$

$$
\begin{aligned}
& i=\underset{l \in\{1, \ldots, K\}}{\arg \max } C_{l}\left(\tilde{P}_{1}^{*}\right) \\
& j=\underset{l \in\{1, \ldots, K\}}{\arg \max } C_{l}\left(\tilde{P}_{2}^{*}\right) .
\end{aligned}
$$

Remark 1: It is important to note that $\mu^{*}$ in (15) and $1-\mu^{*}$ do not directly correspond to the time-sharing factors defined in the optimization problem in (2). In terms of the

\footnotetext{
${ }^{1}$ In the case of multiple maximizers in (16) or (17), any of them can be chosen for the optimal strategy.
} 


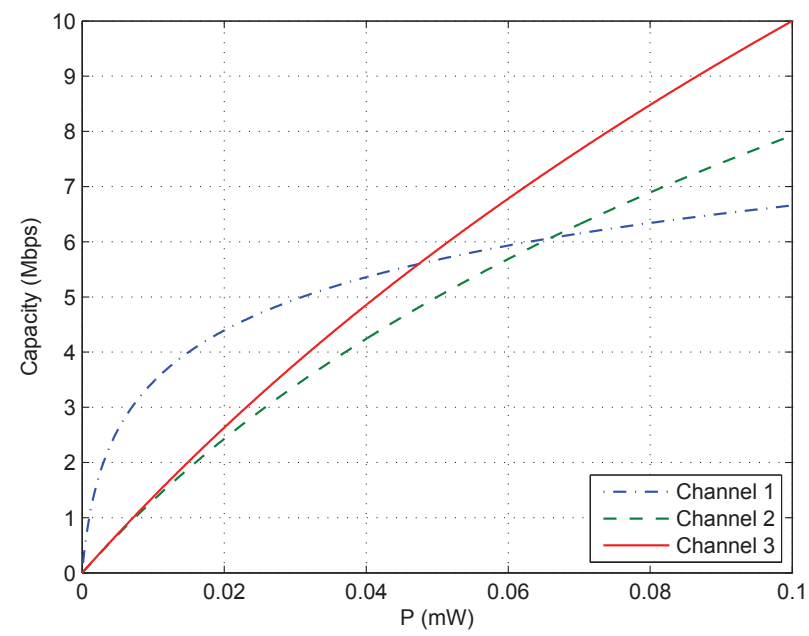

Fig. 3. Capacity of each channel versus power, where $B_{1}=1 \mathrm{MHz}, B_{2}=$ $5 \mathrm{MHz}, B_{3}=10 \mathrm{MHz}, N_{1}=10^{-12} \mathrm{~W} / \mathrm{Hz}, N_{2}=10^{-11} \mathrm{~W} / \mathrm{Hz}$, and $N_{3}=$ $10^{-11} \mathrm{~W} / \mathrm{Hz}$.

notation of the optimization problem in (2), the optimal timesharing factors, denoted by $\lambda_{i}^{*}$ and $\lambda_{j}^{*}$, for the optimal channel switching strategy between channel $i$ and channel $j$ can be obtained based on the transformations in Proposition 1 and Proposition 2 as

$$
\begin{aligned}
& \lambda_{i}^{*}=(1-2 \varepsilon) \mu^{*}+\varepsilon \\
& \lambda_{j}^{*}=(1-2 \varepsilon)\left(1-\mu^{*}\right)+\varepsilon
\end{aligned}
$$

where $\mu^{*}$ is as defined in (15). Since the optimal channel switching strategy is to switch between two channels as stated in Proposition $4, \lambda_{k}^{*}=0$ for $k \in\{1, \ldots, K\} \backslash\{i, j\}$.

Next the solutions of the optimal single channel strategy in (7) and the optimal channel switching strategy in (14) are considered together. Overall, the optimal strategy corresponds to one of them, which achieves the higher average capacity, as expressed in (10).

- If $\varepsilon \geq 1 / 2$, then the optimal single channel strategy outperforms the optimal channel switching strategy since $C_{\mathrm{scs}}$ in (7) always satisfies $C_{\mathrm{scs}}>0$ whereas $C_{\mathrm{css}}=0$ in this case.

- If $\varepsilon<1 / 2$ and $P_{\mathrm{av}} /(1-2 \varepsilon) \geq P_{\mathrm{pk}}$, then the following expressions can be obtained for $C_{\mathrm{scs}}$ :

$$
\begin{aligned}
C_{\mathrm{scs}}=(1-\varepsilon)( & C_{\mathrm{max}}\left(\frac{P_{\mathrm{av}}}{1-\varepsilon}\right) \mathbb{1}_{\left\{\frac{P_{\mathrm{av}}}{1-\varepsilon}<P_{\mathrm{pk}}\right\}} \\
& \left.+C_{\mathrm{max}}\left(P_{\mathrm{pk}}\right) \mathbb{1}_{\left\{\frac{P_{\mathrm{av}}}{1-\varepsilon} \geq P_{\mathrm{pk}}\right\}}\right) \\
>(1-2 \varepsilon) & \left(C_{\max }\left(\frac{P_{\mathrm{av}}}{1-2 \varepsilon}\right) \mathbb{1}_{\left\{\frac{P_{\mathrm{av}}}{1-\varepsilon}<P_{\mathrm{pk}}\right\}}\right. \\
& \left.+C_{\mathrm{max}}\left(P_{\mathrm{pk}}\right) \mathbb{1}_{\left\{\frac{P_{\mathrm{av}}}{1-\varepsilon} \geq P_{\mathrm{pk}}\right\}}\right) \\
\geq(1-2 \varepsilon) & \left(C_{\mathrm{max}}\left(P_{\mathrm{pk}}\right) \mathbb{1}_{\left\{\frac{P_{\mathrm{av}}}{1-\varepsilon}<P_{\mathrm{pk}}\right\}}\right. \\
& \left.+C_{\mathrm{max}}\left(P_{\mathrm{pk}}\right) \mathbb{1}_{\left\{\frac{P_{\mathrm{av}}}{1-\varepsilon} \geq P_{\mathrm{pk}}\right\}}\right) \\
=(1-2 \varepsilon) & C_{\max }\left(P_{\mathrm{pk}}\right)
\end{aligned}
$$

where the equality in (20) is obtained from (7), the inequality in (21) follows from a property of $C_{\max }$ in (12), the relation in (22) is due to the condition $P_{\mathrm{av}} /(1-$ $2 \varepsilon) \geq P_{\mathrm{pk}}$ and the monotone increasing property of $C_{\max }$ in (12), and the final expression in (23) follows from the definition of the indicator function. From (20)-(23), is obtained that $C_{\mathrm{scs}}>(1-2 \varepsilon) C_{\mathrm{max}}\left(P_{\mathrm{pk}}\right)=C_{\mathrm{css}}$; that is, the optimal single channel strategy achieves a higher average capacity than the optimal channel switching strategy for $\varepsilon<1 / 2$ and $P_{\mathrm{av}} /(1-2 \varepsilon) \geq P_{\mathrm{pk}}$.

- Finally, for the case of $\varepsilon<1 / 2$ and $P_{\mathrm{av}} /(1-2 \varepsilon)<P_{\mathrm{pk}}$, the optimal strategy is either the single channel strategy or the channel switching strategy, and the achieved maximum average capacity is expressed as

$$
C_{\mathrm{av}}^{\max }=\max \left(C_{\mathrm{scs}}, C_{\mathrm{css}}\right)
$$

where $C_{\mathrm{scs}}$ is as in (7) and $C_{\mathrm{css}}$ can be calculated as specified in (14), namely,

$$
\begin{gathered}
\max _{\substack{\tilde{P}_{1} \in\left[\frac{P_{\mathrm{av}}}{1-2 \varepsilon}, P_{\mathrm{pk}}\right] \\
\tilde{P}_{2} \in\left[0, \frac{P_{\mathrm{av}}}{1-2 \varepsilon}\right)}}(1-2 \varepsilon) \\
\left(\frac{\frac{P_{\mathrm{av}}}{1-2 \varepsilon}-\tilde{P}_{2}}{\tilde{P}_{1}-\tilde{P}_{2}} C_{\max }\left(\tilde{P}_{1}\right)\right. \\
\left.+\frac{\tilde{P}_{1}-\frac{P_{\mathrm{av}}}{1-2 \varepsilon}}{\tilde{P}_{1}-\tilde{P}_{2}} C_{\max }\left(\tilde{P}_{2}\right)\right) .
\end{gathered}
$$

\section{NumericAl RESUlts}

In this section, numerical examples are presented to investigate the effects of the channel switching delay on the proposed optimal channel switching strategy, and to compare performance of the optimal channel switching and optimal single channel strategies in terms of average capacity maximization. Consider a scenario with $K=3$ channels where the bandwidths and the noise levels (cf. (1)) are given by $B_{1}=$ $1 \mathrm{MHz}, B_{2}=5 \mathrm{MHz}, B_{3}=10 \mathrm{MHz}, N_{1}=10^{-12} \mathrm{~W} / \mathrm{Hz}$, $N_{2}=10^{-11} \mathrm{~W} / \mathrm{Hz}$, and $N_{3}=10^{-11} \mathrm{~W} / \mathrm{Hz}$. Suppose that the peak power constraint and the channel switching delay factor in (2) are set to $P_{\mathrm{pk}}=0.1 \mathrm{~mW}$ and $\varepsilon=0.1$, respectively. In Fig. 3, the capacity of each channel is plotted versus power based on the capacity formula in (1). For the scenario in Fig. 3, the proposed optimal channel switching strategies and the optimal single channel strategy are calculated for various average power limits $\left(P_{\mathrm{av}}\right)$, and the achieved maximum average capacities are plotted versus $P_{\mathrm{av}}$ in Fig. 4. As discussed in the previous section, the optimal single channel strategy achieves a capacity of $(1-\varepsilon) C_{\max }(\phi)$, where $\phi \triangleq \min \left\{\frac{P_{\mathrm{av}}}{(1-\varepsilon)}, P_{\mathrm{pk}}\right\}$ and $C_{\max }(\phi)=\max \left\{C_{1}(\phi), C_{2}(\phi), C_{3}(\phi)\right\}$ in the considered scenario. It is observed from Fig. 3 and Fig. 4 that $C_{\max }(\phi)=$ $C_{1}(\phi)$ for $P_{\mathrm{av}} \in(0,0.0426) \mathrm{mW}$ and $C_{\max }(\phi)=C_{3}(\phi)$ for $P_{\mathrm{av}} \in[0.0426,0.1] \mathrm{mW}$; that is, channel 1 is the best channel up to $P_{\mathrm{av}}=0.0426 \mathrm{~mW}$, and channel 3 is the best after that power level. Among the optimal channel switching strategies discussed in the previous section, it can be observed from Fig. 4 that the optimal channel switching strategy with two channels outperforms the optimal channel switching strategy with three channels for all $P_{\mathrm{av}} \in[0,0.1] \mathrm{mW}$ in accordance with Proposition 4. Overall, the optimal strategy is to employ the optimal channel switching strategy with two channels for 


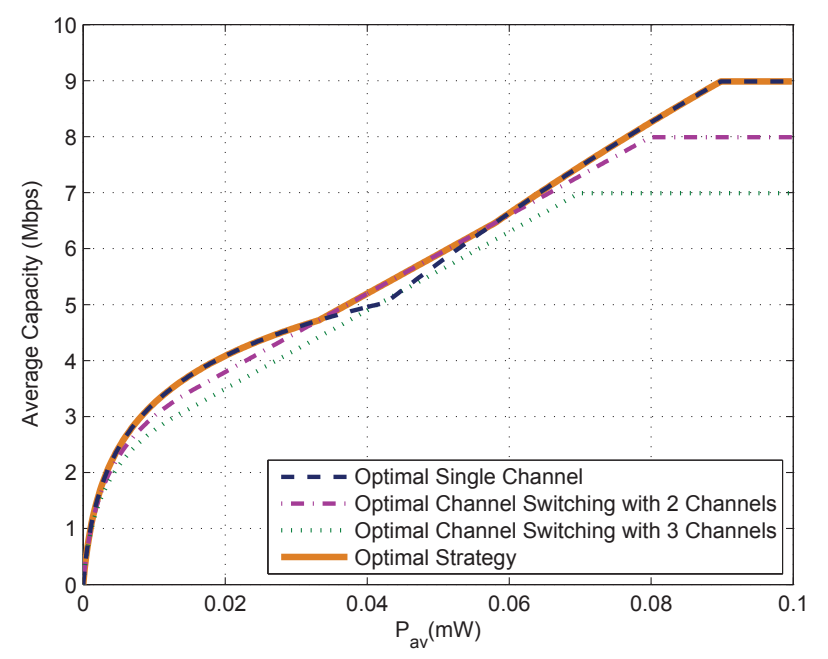

Fig. 4. Average capacity versus average power limit for the optimal channel switching and the optimal single channel strategies for the scenario in Fig. 3, where $P_{\mathrm{pk}}=0.1 \mathrm{~mW}$.

$P_{\text {av }} \in(0.0332,0.0582) \mathrm{mW}$ and the optimal single channel strategy for $P_{\mathrm{av}} \in[0,0.0332] \cup[0.0582,0.1] \mathrm{mW}$. From (14) in Proposition 4, the behaviour of the optimal channel switching strategy with two channels in Fig. 4 can be explained as follows: For $P_{\mathrm{av}} /(1-2 \varepsilon) \geq P_{\mathrm{pk}}$; that is, for $P_{\mathrm{av}} \geq 0.08 \mathrm{~mW}$, $C_{\mathrm{css}}$ in (14) is given by $(1-2 \varepsilon) C_{\max }\left(P_{\mathrm{pk}}\right)=0.8 C_{\max }(0.1)$. On the other hand, for $P_{\mathrm{av}}<0.08 \mathrm{~mW}, C_{\mathrm{css}}$ is calculated from the third expression in (14). In a similar fashion, based on (11) in Proposition 3, the optimal channel switching strategy with three channels achieves an average capacity of $(1-3 \varepsilon) C_{\max }\left(P_{\mathrm{pk}}\right)=0.7 C_{\max }(0.1)$ for $P_{\mathrm{av}} \geq 0.07 \mathrm{~mW}$ and yields the average capacity obtained from the first expression in (11) for $P_{\mathrm{av}}<0.07 \mathrm{~mW}$.

In order to investigate the optimal strategy in Fig. 4 in more detail, Table I presents the solutions of the optimal strategy for various values of the average power limit, $P_{\mathrm{av}}$. In the table, the optimal solution is represented by parameters $\lambda^{*}, P_{1}^{*}, P_{2}^{*}$, $i$, and $j$, meaning that channel $i$ is used with time-sharing factor $\lambda^{*}$ and power $P_{1}^{*}$, and channel $j$ is employed with time-sharing factor $1-\lambda^{*}$ and power $P_{2}^{*}$. From Table I, it is observed that the optimal channel switching strategy with two channels is the optimal strategy for $P_{\mathrm{av}}=0.04 \mathrm{~mW}$ and $P_{\mathrm{av}}=0.05 \mathrm{~mW}$, where switching between channel 1 and channel 3 is performed. For the other $P_{\mathrm{av}}$ values in Table I, it is optimal to employ the optimal single channel strategy.

\section{CONCLUding REMARKS}

In this study, the optimal channel switching problem has been investigated for average capacity maximization in the presence of channel switching delays. First, an equivalent formulation of the optimal channel switching problem has been obtained to facilitate theoretical investigations. Then, the optimal strategy has been obtained and the corresponding average capacity has been specified when channel switching is performed among a given number of channels. Based on this result, it has been shown that optimal channel switching does not involve more than two different channels. Then, the
TABLE I

OPTIMAL STRATEGY FOR THE SCENARIO IN FIG. 3, WHICH EMPLOYS CHANNEL $i$ AND CHANNEL $j$ WITH TIME-SHARING FACTORS $\lambda^{*}$ AND $\left(1-\lambda^{*}\right)$ AND POWER LEVELS $P_{1}^{*}$ AND $P_{2}^{*}$, RESPECTIVELY.

\begin{tabular}{|c||c|c|c||c|c|c|}
\hline$P_{\mathrm{av}}(\mathrm{mW})$ & $\lambda^{*}$ & $P_{1}^{*}$ & $i$ & $\left(1-\lambda^{*}\right)$ & $P_{2}^{*}$ & $j$ \\
\hline 0.01 & - & - & - & 1 & 0.0111 & 1 \\
0.02 & - & - & - & 1 & 0.0222 & 1 \\
0.03 & - & - & - & 1 & 0.0333 & 1 \\
0.04 & 0.4026 & 0.1 & 3 & 0.5974 & 0.0196 & 1 \\
0.05 & 0.527 & 0.1 & 3 & 0.473 & 0.0196 & 1 \\
0.06 & - & - & - & 1 & 0.0667 & 3 \\
0.07 & - & - & - & 1 & 0.0778 & 3 \\
0.08 & - & - & - & 1 & 0.0889 & 3 \\
0.09 & - & - & - & 1 & 0.1 & 3 \\
0.1 & - & - & - & 1 & 0.1 & 3 \\
\hline
\end{tabular}

scenarios under which the optimal strategy corresponds to the exclusive use of a single channel or to channel switching between two channels have been specified. Via numerical examples, the theoretical results and the effects of channel switching delays have been illustrated.

\section{REFERENCES}

[1] R. G. Gallager, Information Theory and Reliable Communication. New York, NY, USA: John Wiley \& Sons, Inc., 1968.

[2] L. Li and A. Goldsmith, "Capacity and optimal resource allocation for fading broadcast channels- Part I: Ergodic capacity," IEEE Trans. Inf. Theory, vol. 47, no. 3, pp. 1083-1102, Mar. 2001.

[3] Z. Shen, J. Andrews, and B. L. Evans, "Adaptive resource allocation in multiuser OFDM systems with proportional rate constraints," IEEE Trans. Wireless Commun., vol. 4, no. 6, pp. 2726-2737, Nov. 2005.

[4] M. Azizoglu, "Convexity properties in binary detection problems," IEEE Trans. Inform. Theory, vol. 42, no. 4, pp. 1316-1321, July 1996.

[5] Y. Ma and C. C. Chai, "Unified error probability analysis for generalized selection combining in Nakagami fading channels," IEEE J. Select. Areas Commun., vol. 18, no. 11, pp. 2198-2210, Nov. 2000.

[6] J. A. Ritcey and M. Azizoglu, "Performance analysis of generalized selection combining with switching constraints," IEEE Commun. Letters, vol. 4, no. 5, pp. 152-154, May 2000.

[7] B. Dulek, M. E. Tutay, S. Gezici, and P. K. Varshney, "Optimal signaling and detector design for M-ary communication systems in the presence of multiple additive noise channels," Digital Signal Processing, vol. 26, pp. 153-168, Mar. 2014

[8] M. E. Tutay, S. Gezici, H. Soganci, and O. Arikan, "Optimal channel switching over Gaussian channels under average power and cost constraints," IEEE Trans. Commun., 2015.

[9] A. D. Sezer, S. Gezici, and H. Inaltekin, "Optimal channel switching strategy for average capacity maximization," IEEE Trans. Commun., vol. 63, no. 6, pp. 2143-2157, June 2015.

[10] P. Bahl, R. Chandra, and J. Dunagan, "SSCH: Slotted seeded channel hopping for capacity improvement in IEEE 802.11 ad-hoc wireless networks," in Proc. Annual Int. Conf. on Mobile Computing and Networking (Mobicom), 2004, pp. 216-230.

[11] S. Krishnamurthy, M. Thoppian, S. Venkatesan, and R. Prakash, "Control channel based MAC-layer configuration, routing and situation awareness for cognitive radio networks," in Proc. IEEE Military Commun. Conf. (MILCOM), vol. 1, Oct. 2005, pp. 455-460.

[12] M. Yun, Y. Zhou, A. Arora, and H.-A. Choi, "Channel-assignment and scheduling in wireless mesh networks considering switching overhead," in Proc. IEEE Int. Conf. on Commun. (ICC), June 2009, pp. 1-6.

[13] T. M. Cover and J. A. Thomas, Elements of Information Theory. WileyInterscience, 1991 